\title{
A murine model of dry eye induced by topical administration of erlotinib eye drops
}

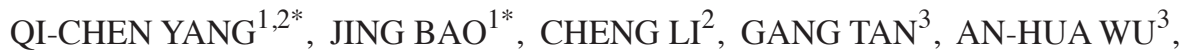 \\ LEI YE ${ }^{1}$, LIN-HONG YE ${ }^{1}$, QIONG ZHOU ${ }^{1}$ and YI SHAO ${ }^{1}$ \\ ${ }^{1}$ Department of Ophthalmology, The First Affiliated Hospital of Nanchang University, Nanchang, Jiangxi 330006; \\ ${ }^{2}$ Eye Institute of Xiamen University, Fujian Provincial Key Laboratory of Ophthalmology and Visual Science, \\ Xiamen, Fujian 361102; ${ }^{3}$ Department of Ophthalmology, The First Affiliated Hospital \\ of The University of South China, Hengyan, Hunan 421001, P.R. China
}

Received March 18, 2017; Accepted December 19, 2017

DOI: $10.3892 /$ ijmm.2017.3353

\begin{abstract}
In the present study, the effects of erlotinib on mouse tear function and corneal epithelial tissue structure were investigated. Throughout the 3 weeks of treatment, no notable differences were observed in the body, eye or lacrimal gland weights of the control and experimental mice. However, in the experimental group, the tear volume and break-up times of tear film were significantly lower following treatment with erlotinib compared with the control group. Corneal fluorescein staining in the experimental group revealed patchy staining, and the Lissamine green staining and inflammatory index were significantly higher in the experimental group at 3 weeks than in the control group. In the experimental group, the number of corneal epithelium layers increased significantly following treatment with erlotinib for 3 weeks and a significant increase in the number of vacuoles was observed compared with the control group. Treatment with erlotinib significantly increased the corneal epithelial cell apoptosis, and led to a significantly increased number of epithelial cell layers and increased keratin 10 expression. It also significantly reduced the number of conjunctival goblet cells. Transmission electron microscopy and scanning electron microscopy revealed that the corneal epithelial surface was irregular and there was a substantial reduction and partial loss of the microvilli in the experimental group. Mice treated with erlotinib also exhibited an increased protein expression of tumor necrosis factor- $\alpha$ and decreased protein expression of phosphorylated-epidermal growth factor
\end{abstract}

Correspondence to: Professor Yi Shao, Department of Ophthalmology, The First Affiliated Hospital of Nanchang University, 17 Yongwaizheng Street, Nanchang, Jiangxi 330006, P.R. China

E-mail: freebee99@163.com

${ }^{*}$ Contributed equally

Key words: erlotinib, ocular surface, dry eye, mouse receptor in the corneal epithelial cells. The topical application of erlotinib eye drops was revealed to induce dry eyes in mice. This is a novel method of developing a model of dry eyes in mice.

\section{Introduction}

The incidence of lung cancer is increasing each year, this is largely due to increased levels of pollution and bad habits, such as smoking (1). In total, 80-85\% of lung cancer cases are non-small cell lung cancer (NSCLC), and $>70 \%$ of patients are at a locally advanced or late stage of the disease when they are diagnosed (2). Erlotinib is a small molecule that acts as an epidermal growth factor receptor (EGFR) tyrosine kinase inhibitor (3). Erlotinib is a drug used for the second-line treatment of patients with locally advanced or metastatic NSCLC (4). Erlotinib binds to EGFR with high specificity (5). A previous study has revealed that erlotinib may hinder the growth of retinoblastoma by inhibiting the tyrosine kinase activity of the EGFR intracellular domain, thereby inhibiting cell proliferation and angiogenesis, and inducing the apoptosis of tumor cells (6). There are various side effects of erlotinib, the most common are a rash, abdominal pain, nausea, vomiting and headache (7). Previous studies have also reported that one of the side effects of erlotinib is dry eyes (8). A study by Fraunfelder et al (9) proposed that erlotinib may cause or aggravate dry eyes. Johnson et al (10) reported non-healing of corneal erosion and infectious keratitis cases caused by the use of erlotinib for the treatment of lung tumors. Morishige et al (11) also reported a case of diffuse water deficiency dry eye following treatment with erlotinib. Patients have also been reported to exhibit corneal dissolution and perforation following treatment with erlotinib (12). In the present study, it was revealed that erlotinib may cause many of the symptoms of dry eyes following its topical administration in mice.

\section{Materials and methods}

Animal preparation. A total of 60 male specific pathogen-free BALB/c mice (age, 6-8 weeks; weight, 18-20 g) were purchased 
from the Laboratory Animal Center of Xi'an Jiao Tong University College of Medicine (Xi'an, China) and used in the present study. All mice had free asses to food and water. No abnormalities were identified in the anterior segment or fundus of the eyes when they underwent slit-lamp microscopy and fundus examination. The Schirmer I test results were $\geq 10 \mathrm{~mm} / 5 \mathrm{~min}$ (13). The mice were kept in a standard housing environment throughout the study, with a room temperature of $\sim 25 \pm 1^{\circ} \mathrm{C}$, relative humidity $\sim 60 \pm 10 \%$ and an alternating 12-h light/dark cycle, as previously described (14). All procedures were performed in accordance with the Association for Research in Vision and Ophthalmology Statement for the Use of Animals in Ophthalmic and Vision Research and the present study was approved by the Medical and Animal Ethics Committee of The First Affiliated Hospital of Nanchang University (Nanchang, China).

Preparation of erlotinib eye drops. To prepare the eye drops, erlotinib (Tarceva ${ }^{\circledR}$; Roche Diagnostics, Indianapolis, IN, USA) was diluted in sterile PBS to a concentration of $20 \mu \mathrm{M}$ and homogenized by ultrasound vortex $\left(37^{\circ} \mathrm{C}, 100 \mathrm{~W}, 10 \mathrm{~min}\right)$. The preservative benzyl bromide (Sigma-Aldrich; Merck KGaA, Darmstadt, Germany) was added to the two groups (with a concentration controlled at $0.005 \%$ ); one group was a control group using PBS eye drops, and the second group was the experimental group that received erlotinib eye drops. Eye drops were stored at $4^{\circ} \mathrm{C}$ prior to their use.

Animal experimental procedure. The 60 mice were divided into two groups. Group $1(\mathrm{n}=30)$ received PBS treatment and group $2(n=30)$ received erlotinib treatment. All mice received $5 \mu 1$ of the respective eye drops in their right eye, four times daily. Prior to treatment, all mice were free from ocular diseases. Prior to treatment and at 1-, 2- and 3-weeks post treatment, a Schirmer test, fluorescein staining, break-up time (BUT) of tear film, Lissamine ${ }^{\mathrm{TM}}$ green staining and hematoxylin and eosin (H\&E) staining were performed on each group. Following 3 weeks of treatment, the eyeballs were enucleated and processed for light and electron microscopy analyses of the structural changes to the corneal epithelial cells. Periodic acid-Schiff (PAS) staining was performed to visualize changes to conjunctival goblet cells. Histological sections were used for the detection of keratin (K)10 and apoptotic cells. Tumor necrosis factor (TNF)- $\alpha$, phosphorylated (p)-EGFR and EGFR protein were detected by western blot analysis.

Fluorescein and BUT. A total of $1 \mu 10.1 \%$ liquid sodium fluorescein was applied onto the conjunctival sac. Following three blinks, BUTs were recorded in sec. After $90 \mathrm{sec}$, the corneal epithelial damage was graded using a cobalt blue filter under a slit-lamp microscope (Chongqing Kanghua Ruiming S\&T Co., Ltd., Chongqing, China) with a reticule calibrated for x16 magnification. The cornea was divided into four quadrants and they were scored individually. The fluorescein score was analyzed as previously described (15) with essential modifications as follows: Absent, 0 ; slightly punctate staining $\leq 30$ spots, 1 ; punctate staining $>30$ spots but not diffuse, 2; severe diffuse staining but no positive plaque, 3 ; positive fluorescein plaque, 4 . The scores of each quadrant were added together to give a final score (maximum, 16 points).
Corneal dye staining. To evaluate changes in the corneal epithelial cells, one drop of 3\% Lissamine Green B (Sigma-Aldrich; Merck KGaA) was applied onto the inferior lateral conjunctival sac. The corneal surface was observed using a slit-lamp microscope with a reticule calibrated for x16 magnification and the staining of the cornea was scored in a blinded manner as follows: Score 0 for no punctuate staining; score 1 when $<1 / 3$ of the cornea was stained; score 2 when $\leq 2 / 3$ was stained; and score 3 when $>2 / 3$ was stained (16).

PAS staining. The whole eyeball, including the superior and inferior forniceal conjunctiva, was excised and fixed in $4 \%$ formalin for $12 \mathrm{~h}$ at $4^{\circ} \mathrm{C}$. The tissue was then cut into $4-\mu \mathrm{m}$ thick sections through the superior and inferior conjunctival fornices and stained with PAS (Sigma-Aldrich; Merck KGaA) at room temperature. Briefly, $4-\mu \mathrm{m}$ slices were treated with $0.5 \%$ periodic acid for $10 \mathrm{~min}$, and then saturated in dimedone aqueous solution for $10 \mathrm{~min}$. The slices were then incubated with Schiff's reagent for $8 \mathrm{~min}$ at room temperature. The nuclei were stained with hematoxylin for $30 \mathrm{sec}$. The number of PAS-stained cells was counted per $100 \mathrm{~mm}^{2}$ in four sections of the eye from each mouse, the average count was determined in each eye as the goblet cell density. The count was performed by the same observer each time, as previously described (15). Corneal tissues were stained with $\mathrm{H} \& \mathrm{E}$ at room temperature. All images were captured by a light microscope (Carl Zeiss AG, Oberkochen, Germany) with a magnification of x 20 .

Evaluation of inflammation. The inflammatory response was evaluated by slit-lamp microscopy with a reticule calibrated for x16 magnification at 9:00 am on the first day of weeks 0, 1,2 and 3 . The inflammatory index was analyzed as previously described (16). Briefly, the inflammatory index was scored based on the following parameters: Ciliary hyperemia (absent, 0 ; present but $<1 \mathrm{~mm}, 1$; present $1-2 \mathrm{~mm}, 2$; and present $>2 \mathrm{~mm}, 3$ ); central corneal edema (absent, 0; present with visible iris details, 1 ; present without visible iris details, 2 ; and present without visible pupil, 3); and peripheral corneal edema (absent, 0; present with visible iris details, 1; present without visible iris details, 2; and present with no visible iris, 3). The final inflammatory index result was obtained by totaling the scores for the different parameters and dividing them by a factor of nine.

Terminal deoxynucleotidyl transferase mediated dUTP biotin nick end labeling (TUNEL) assay. A TUNEL assay was performed according to a previously published method, with some modifications (17). Eyeballs were fixed in $4 \%$ buffered formalin for $24 \mathrm{~h}$ at $4^{\circ} \mathrm{C}$ and then paraffin-embedded. Sections (5 $\mu \mathrm{m}$ thick) were pre-coated in Histogrip (Zymed; Thermo Fisher Scientific, Inc., Waltham, MA, USA) in acetone. The dilution of Histogrip was 1:50. Subsequently, the sections were permeabilized with $4 \mu \mathrm{g} / \mathrm{ml}$ proteinase $\mathrm{K}$ (Merck KGaA) for $10 \mathrm{~min}$ at room temperature. Following this, sections were incubated with terminal deoxynucleotidyl transferase in buffer containing cobalt chloride, potassium cacodylate, Tris- $\mathrm{HCl}$, bovine serum albumin (BSA), biotinylated deoxy-uridine triphosphate (dUTP), and deoxy-adenosine triphosphate (Boehringer-Mannheim $\mathrm{GmbH}$, Mannheim, Germany) for $60 \mathrm{~min}$ at $37^{\circ} \mathrm{C}$. The reaction was terminated by incubation 
with sodium chloride/sodium citrate buffer for $15 \mathrm{~min}$ at room temperature, in PBS for $1 \mathrm{~min}$, and in PBS containing FCS and Triton X-100 (Sigma-Aldrich; Merck KGaA) for 30 min at room temperature. Sections were washed in PBS for $10 \mathrm{~min}$, and then incubated with horseradish peroxidase-conjugated streptavidin (1:300; P0397; Dako; Agilent Technologies, Inc., Santa Clara, CA, USA) in PBS for $1 \mathrm{~h}$ at room temperature. Negative control sections were stained identically, but with omission of biotinylated dUTP from the nick end labelling mixture (17). Apoptotic cells in tissue were counted in a $1-\mathrm{mm}^{2}$ area of epithelium in each section, three sections from each sample were counted by light microscopy (TE-200-OU; Nikon Corporation, Tokyo, Japan).

Immunofluorescent staining of K10. Immunodetection of K10 was performed as described previously (15).Immunofluorescent staining was performed in cryosections $(6-\mu \mathrm{m}$ thick) of the eyeballs. Sections were fixed in $99.5 \%$ acetone (Sinopharm Chemical Reagent Co., Ltd., Shanghai, China) at $-20^{\circ} \mathrm{C}$ for $10 \mathrm{~min}, 2 \% \mathrm{BSA}$ for blocking, and then incubated at $4^{\circ} \mathrm{C}$ overnight with K10 antibody. Mouse anti-human K10 antibodies (ab16667; Abcam, Cambridge, UK) were used at a dilution of 1:150 as the primary antibodies, followed by Alexa Fluor ${ }^{\circledR}$ secondary goat anti-mouse immunoglobulin (Ig)G (1:300; A-11001; Invitrogen; Thermo Fisher Scientific Inc.) incubation at room temperature for $1 \mathrm{~h}$. Nuclei were counterstained with $0.5 \mathrm{~g} / \mathrm{ml}$ Hoechst 33342 dye (Thermo Fisher Scientific Inc.). Subsequently, the specimens were observed under a fluorescent microscope with a magnification of x20 (Zeiss GmbH, Jena, Germany).

Scanning electron microscopy. On day 21, the corneas were fixed in $2.5 \%$ glutaraldehyde in $0.1 \mathrm{M}$ phosphate buffer $(\mathrm{pH} 7.4)$ for $24 \mathrm{~h}$ at $4^{\circ} \mathrm{C}$. Specimens were subsequently post-fixed with $1 \%$ osmium tetroxide in $0.1 \mathrm{M}$ phosphate buffer for $2 \mathrm{~h}$ and dehydrated in a graded series of ethanol solutions at $4^{\circ} \mathrm{C}$. Following dehydration, the fixed specimens were critical-point dried, gold coated with platinum, and examined with a scanning electronic microscope with a magnification of $\mathrm{x} 10,000$ (JSM-6330F; JEOL, Ltd., Tokyo, Japan).

Transmission electron microscopy (TEM). On day 21 following erlotinib treatment, the right corneas were harvested and fixed for $2 \mathrm{~h}$ in $4 \%$ glutaraldehyde $(\mathrm{pH}=7.4)$ at $37^{\circ} \mathrm{C}$, washed with $1 / 15 \mathrm{M}$ phosphate buffer, post-fixed in osmium tetroxide for $2 \mathrm{~h}$ at room temperature, washed again, and dehydrated in an acetone series. The specimens were embedded using epoxy resin in accordance with the standard method. The embedding blocks were sliced to $50-\mathrm{nm}$ sections. Following baking and dyeing using uranyl acetate-lead citrate staining for $1 \mathrm{~h}$ at room temperature, the ultrastructure of the corneal epithelial layer was examined and captured using TEM with a magnification of x10,000 (model no. H7650; Hitachi, Tokyo, Japan). Microvilli in epithelium tissue were counted in each photo, and three photos from each sample were counted.

Western blotting. The cornea and conjunctiva were lysed with cold radioimmunoprecipitation buffer (1\% Triton X-100, $1 \%$ sodium deoxycholate. $0.1 \%$ sodium dodecyl sulfate, $0.15 \mathrm{M} \mathrm{NaCl}, 0.05 \mathrm{M}$ Tris- $\mathrm{HCl}$ ). Protein concentrations were measured using a BCA kit (MicroBCA; Pierce; Thermo Fisher Scientific, Inc.). Equal amounts $(20 \mu \mathrm{l})$ of proteins were subjected to electrophoresis on $8 \%$ SDS-PAGE and then transferred into polyvinylidene difluoride membranes. The membranes were blocked with $2 \%$ BSA at room temperature for $1 \mathrm{~h}$ and then incubated with primary antibodies directed against EGFR (1:1,000; ab52894), p-EGFR (1:1,000; ab40815) or TNF- $\alpha$ (1:400; ab183218) (all from Abcam, Cambridge, MA, USA) and $\beta$-actin (1:10,000; A5441; Sigma-Aldrich; Merck $\mathrm{KGaA}$ ) as a loading control at $4^{\circ} \mathrm{C}$ overnight, as previously described (15). The membranes were subsequently incubated with horseradish peroxidase-conjugated goat anti-rabbit IgG (1:10,000; 1706515; Bio-Rad Laboratories, Inc., Hercules, CA, USA) secondary antibodies at room temperature for $2 \mathrm{~h}$. Signals were developed using enhanced chemiluminescence reagents (Xiamen Lulong Biotech Co., Ltd., Xiamen, China) and captured on film.

Statistical analysis. Data were presented as the mean \pm standard deviation. Statistical analyses were performed using SPSS version 16.0.0 (SPSS, Inc., Chicago, IL, USA). One-way analysis of variance and Bonferroni's post hoc tests were applied in all comparisons between groups. $\mathrm{P}<0.05$ was considered to indicate a statistically significant difference.

\section{Results}

Metabolic conditions of treatment mice. Body, eyeball and extraorbital lacrimal gland weights were measured in each group at $0,1,2$, and 3 weeks following the start of treatment with either $20 \mu \mathrm{M}$ erlotinib or PBS (Fig. 1A). No significant differences were identified in body, eyeball or lacrimal gland weights between the experimental erlotinib group and the PBS control group (Fig. 1B-D, respectively).

Erlotinib treatment causes tear film damage and significantly decreases BUT and tear volume. At 3 weeks following the commencement of treatment, tear film and epithelium damage were observed in the erlotinib group (Fig. 2A). This was possibly due to the toxicity of the erlotinib. Following 3 weeks of treatment, PBS-treated cornea did not have fluorescein sodium and Lissamine green staining. The fluorescein sodium scores (Fig. 2B) and Lissamine green staining (Fig. 2C) were significantly increased in the erlotinib group at all time points following treatment compared with that in the PBS group $(\mathrm{P}<0.05)$. Prior to treatment, no significant differences were identified in BUTs, tear volume or scores of tear film, or epithelium damage between the two groups. At 1, 2 and 3 weeks following treatment, the erlotinib group demonstrated significantly decreased BUTs and tear volume compared with that observed in the PBS group $(\mathrm{P}<0.05$; Fig. 2D and $\mathrm{E})$.

Erlotinib treatment increases the inflammatory index. There was a significant increase in the inflammatory index at all time points following the commencement of treatment in the erlotinib-treated group compared with that observed in the PBS-treated group $(\mathrm{P}<0.05 ;$ Fig. 2F).

Erlotinib treatment leads to structural changes in the epithelium. The number of epithelial layers in the central 
A

Group $1(n=30)$

Group $2(n=30)$
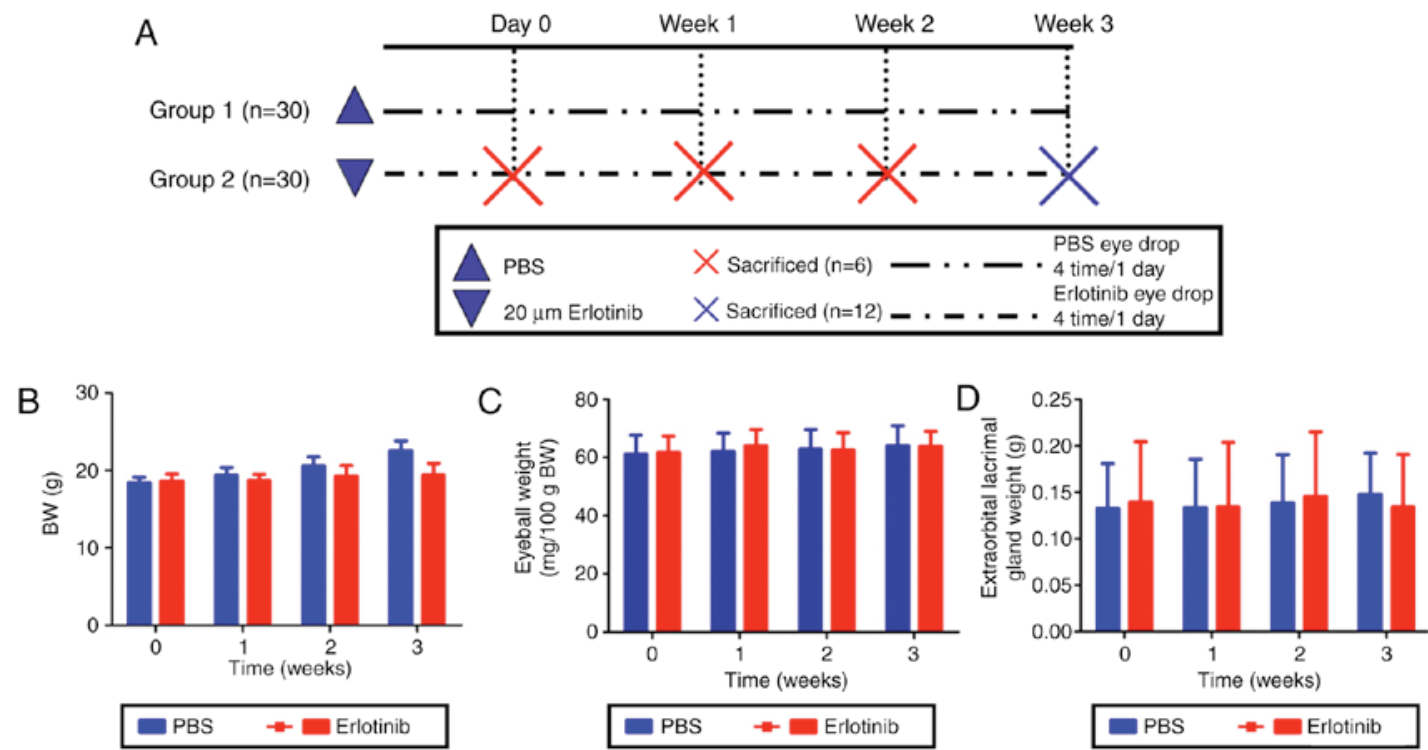

Figure 1. Experimental design. (A) A total of 60 mice were randomized into two groups (n=30/group). Group 1 were administered PBS eye drops, 4 times/day, whereas group 2 were administered $20 \mu \mathrm{M}$ erlotinib 4 times/day. The (B) body, (C) eyeball and (D) extraorbital lacrimal gland weights of the mice were measured at the indicated time points. Data are presented as the mean \pm standard deviation. BW, body weight.
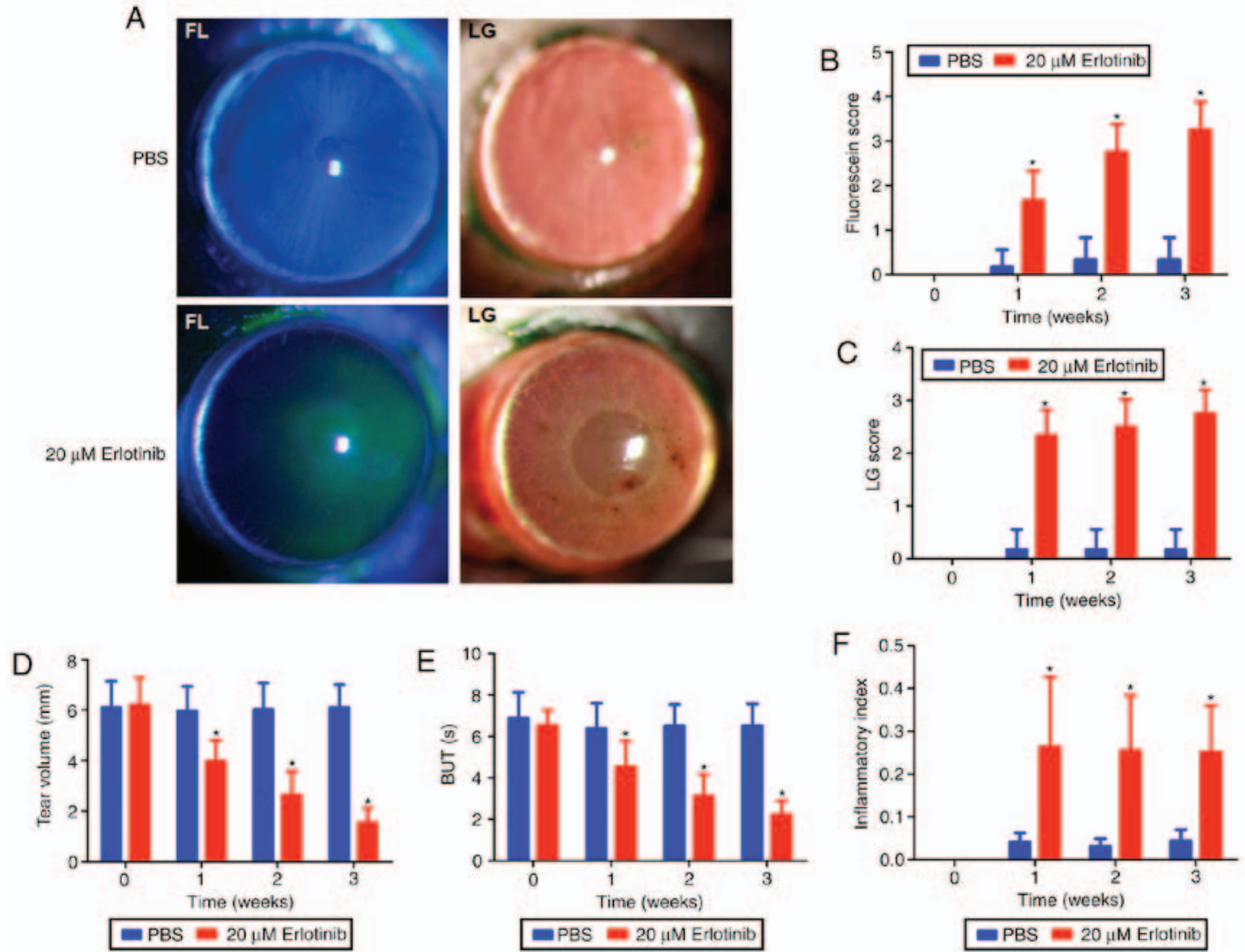

Figure 2. Alterations to the ocular surface and degree of inflammation following treatment with erlotinib. (A) Representative examples of FL and LG staining following 3 weeks of treatment with either erlotinib or PBS. Images were captured using a slit-lamp microscope with a reticule calibrated for x16 magnification. The (B) corneal fluorescein staining score and the (C) LG scores were measured at the indicated time points. The (D) tear volume and (E) BUTs of the ocular surface were also recorded at the indicated time points in each group. (F) The overall inflammatory index for each group was determined. " $\mathrm{P}<0.05 \mathrm{vs}$. the PBS group at the same time point. Data are presented as the mean \pm standard deviation. BUT, break-up time. FL, fluorescein sodium; LG, Lissamine green.

cornea was significantly increased in the erlotinib group compared with the number in the PBS group following 3 weeks of treatment (Fig. 3A and B; $\mathrm{P}<0.05$ ). A significant increase in the number of corneal vacuole cells was also observed in the erlotinib group compared with the number in the PBS group ( $\mathrm{P}<0.05$; Fig. $3 \mathrm{C})$. 

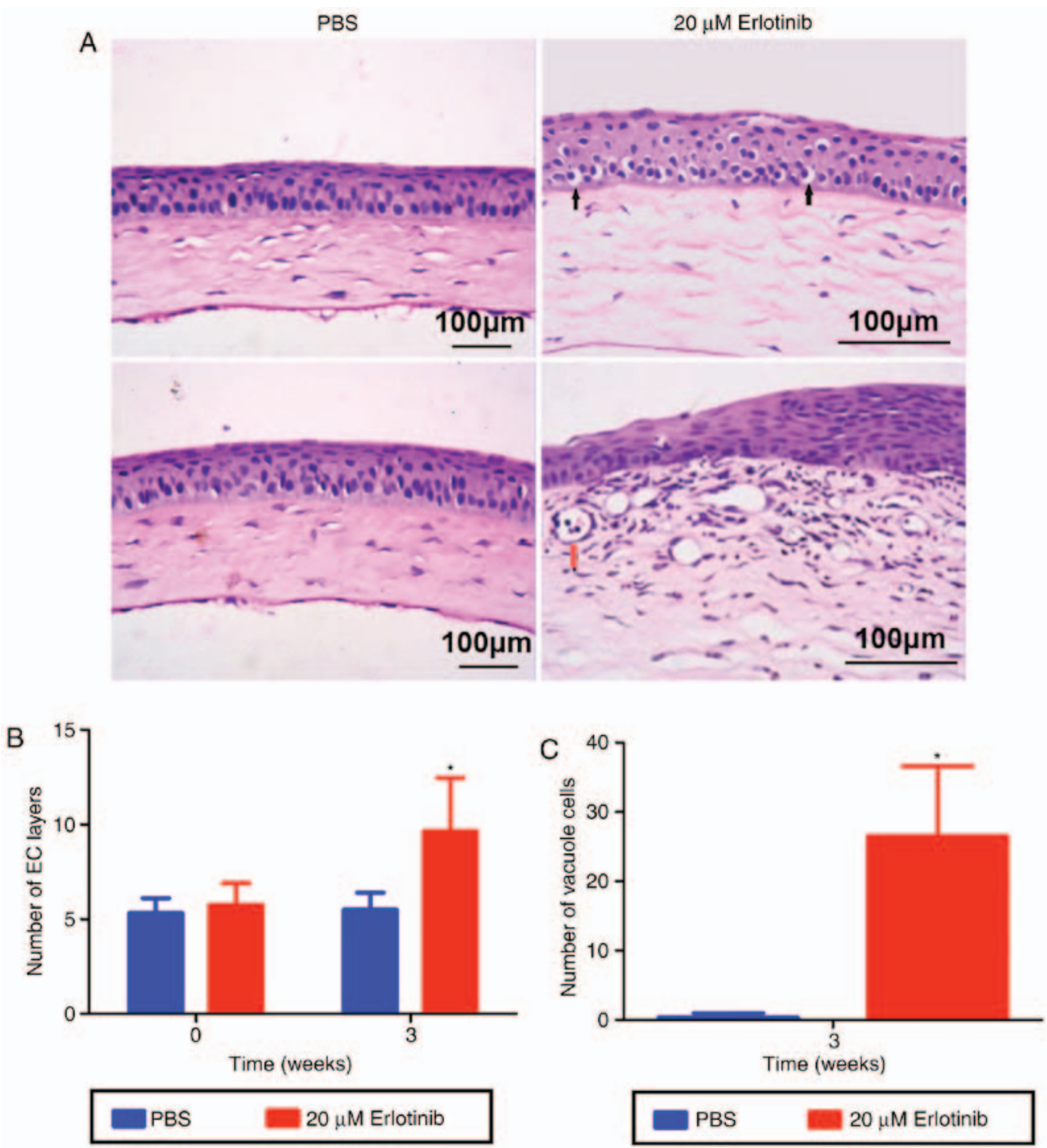

Figure 3. Alterations to the corneal epithelium following treatment. (A) Representative images demonstrating additional EC layers vacuole cells (black arrows) and new vessels (red arrows) in the cornea following treatment with erlotinib. (B) The number of EC layers and (C) the number of vacuole cells in the cornea epithelium was determined. Scale bar, $100 \mu \mathrm{m}$. Stained with hematoxylin and eosin. ${ }^{*} \mathrm{P}<0.05$ vs. the PBS group at the same time point. EC, epithelial cell.

Erlotinib treatment causes a reduction in goblet cell number. PAS staining was used to examine the effect of erlotinib on goblet cells in the conjunctiva (Fig. 4A). The results revealed that the PAS-positive cell number was significantly decreased in the erlotinib group at 3 weeks compared with the number in the PBS group ( $\mathrm{P}<0.05$; Fig. 4B). No notable difference was observed in the number of goblet cells in the PBS group at 3 weeks compared with the beginning of treatment.

Erlotinib treatment causes a significant increase in apoptosis. Compared with the PBS group, immunostaining of K10 revealed a notable increase in K10-positive cells in the central cornea of the erlotinib group following 3 weeks of treatment (Fig. 5A). A TUNEL assay revealed that apoptosis was induced in the corneal superficial but not in the stroma of the erlotinib-treated group. At 3 weeks following the commencement of treatment, the number of apoptotic cells and level of K10 were significantly increased in the corneal epithelium of the erlotinib-treated group compared with the level in the PBS-treated group ( $\mathrm{P}<0.05$; Fig. 5B).
Erlotinib treatment causes ultrastructural changes to the corneal epithelium. The cornea epithelium was intact and well organized in the PBS group following 3 weeks of treatment (Fig. 5C). In contrast, the epithelial cells in the cornea of the erlotinib group were deformed following 3 weeks of treatment. TEM revealed enriched regularly arranged microvilli and desmosomes extending from surface epithelial cells in the PBS group following 3 weeks of treatment; whereas, in the erlotinib group, the majority of microvilli were much shorter and disorganized, and the morphology of microvilli was also clearly different from the PBS group. The number of corneal epithelial microvilli was significantly reduced in the erlotinib group compared to the number in the PBS group following 3 weeks of treatment $(\mathrm{P}<0.05$; Fig. 5D).

Inflammatory changes of the ocular surface. To investigate the mechanisms underlying erlotinib-induced damage, the expression of TNF- $\alpha$, p-EGFR and EGFR was examined by western blot analysis. The results revealed that, compared with the PBS group, TNF- $\alpha$ protein levels were significantly higher in the 
A

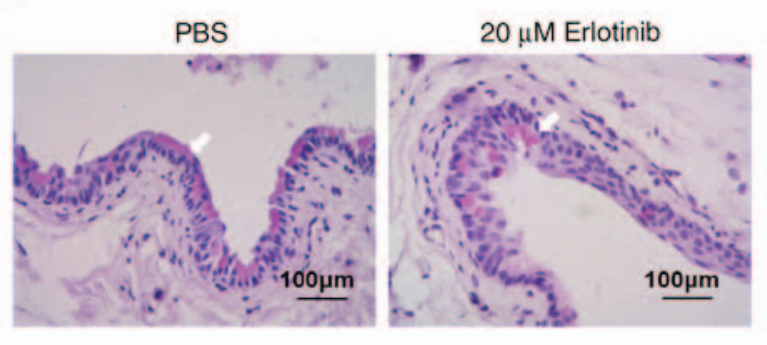

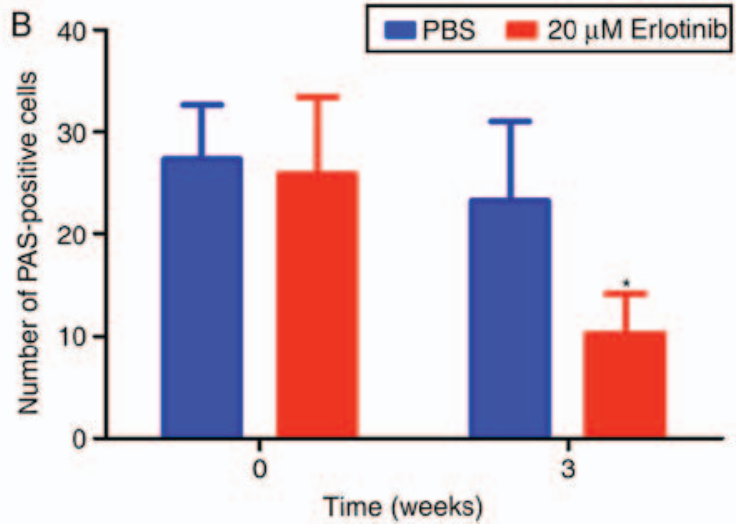

Figure 4. Representative images for PAS staining in the conjunctiva. (A) PAS staining of the forniceal conjunctiva following 3 weeks of treatment with either PBS or $20 \mu \mathrm{M}$ erlotinib. The goblet cells (white arrows) were abundantly present in the conjunctival fornix of the PBS-treated eyes but decreased following treatment with erlotinib. (B) The average number of PAS-positive cells in the conjunctiva was determined. The number was significantly lower in the erlotinib group compared with the PBS group following 3 weeks of treatment. Scale bar, $100 \mu \mathrm{m} .{ }^{*} \mathrm{P}<0.05$ vs. the PBS group at the same time point. Data are presented as the mean \pm standard deviation. PAS, periodic acid-Schiff.
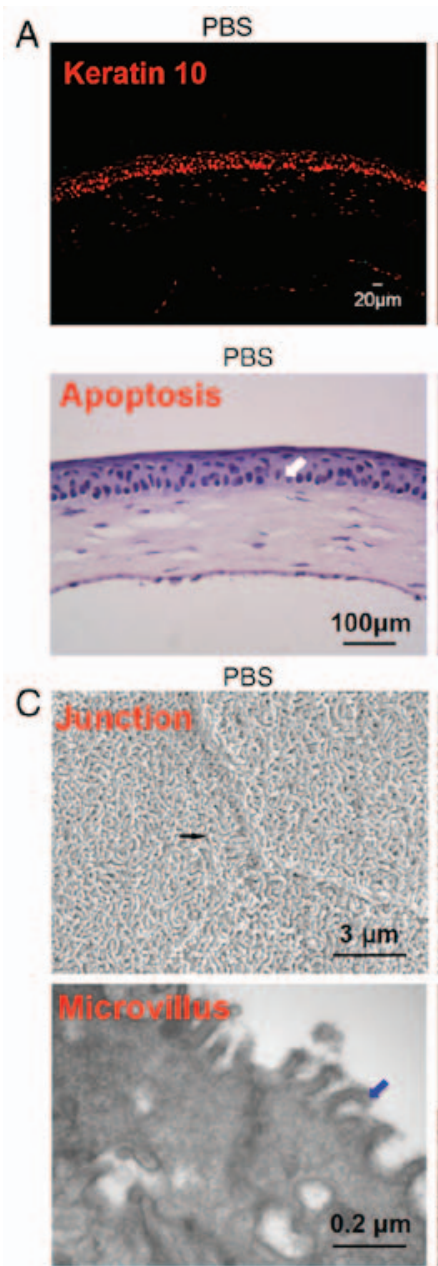

$20 \mu \mathrm{M}$ Erlotinib

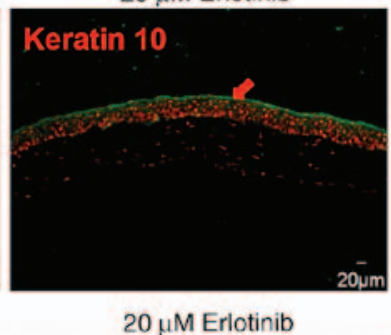

Apoptosis

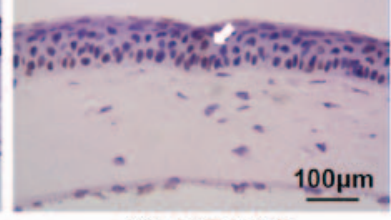

$20 \mu \mathrm{M}$ Erlotinib
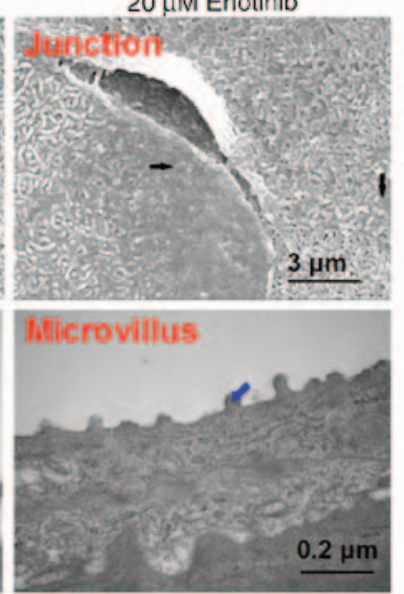
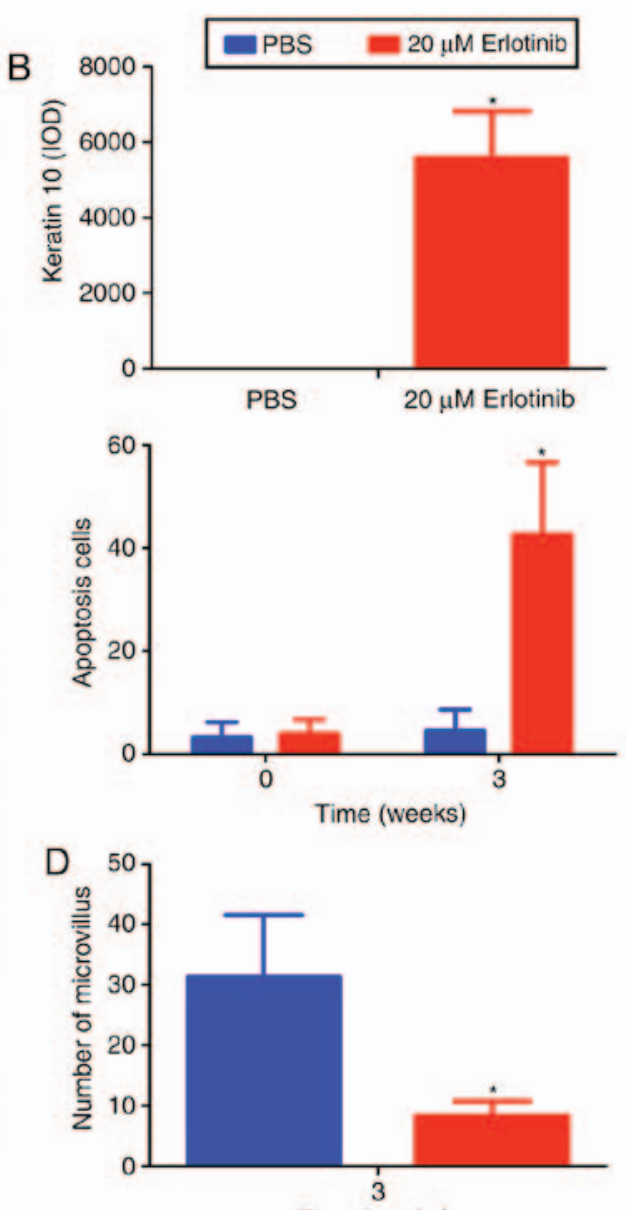

Time (weeks)

- $20 \mu \mathrm{M}$ Erlotinib

Figure 5. Corneal epithelial squamous metaplasia ultrastructure. (A) Representative images from a keratin 10 (green line and red arrow) and a terminal deoxynucleotidyl transferase mediated dUTP biotin nick end labeling assay of the cornea epithelium following 3 weeks of treatment with either PBS or erlotinib. White arrows indicate apoptosis-positive cells. (B) The level of apoptosis and keratin 10 was quantified. Few apoptotic cells were observed in the superficial layer of the corneal epithelium in the PBS group, while significantly more apoptosis was recorded in the corneal superficial and basal epithelium following treatment with erlotinib. Compared with the PBS group, there was a significant upregulation of keratin 10-positive cells in the central cornea of the erlotinib group following 3 weeks of treatment. (C) In the PBS group, an integrated junction between epithelial cells was observed by scanning electron microscopy (upper left image, black arrow), whereas a destroyed junction (upper right image, black arrow) was observed in the erlotinib group. Following treatment with PBS for 3 weeks, the corneal epithelial microvilli were extended as digitations and arranged neatly (lower left image, blue arrow). Following treatment with erlotinib for 3 weeks, the corneal epithelial microvilli were shorter and disordered (lower right image, blue arrow). (D) The number of microvilli observed in the superficial layer of the corneal epithelium was calculated for each group. Data are presented as the mean + standard deviation. " $\mathrm{P}<0.05$ vs. the PBS group at the same time point. IOD, integrated optical density. 

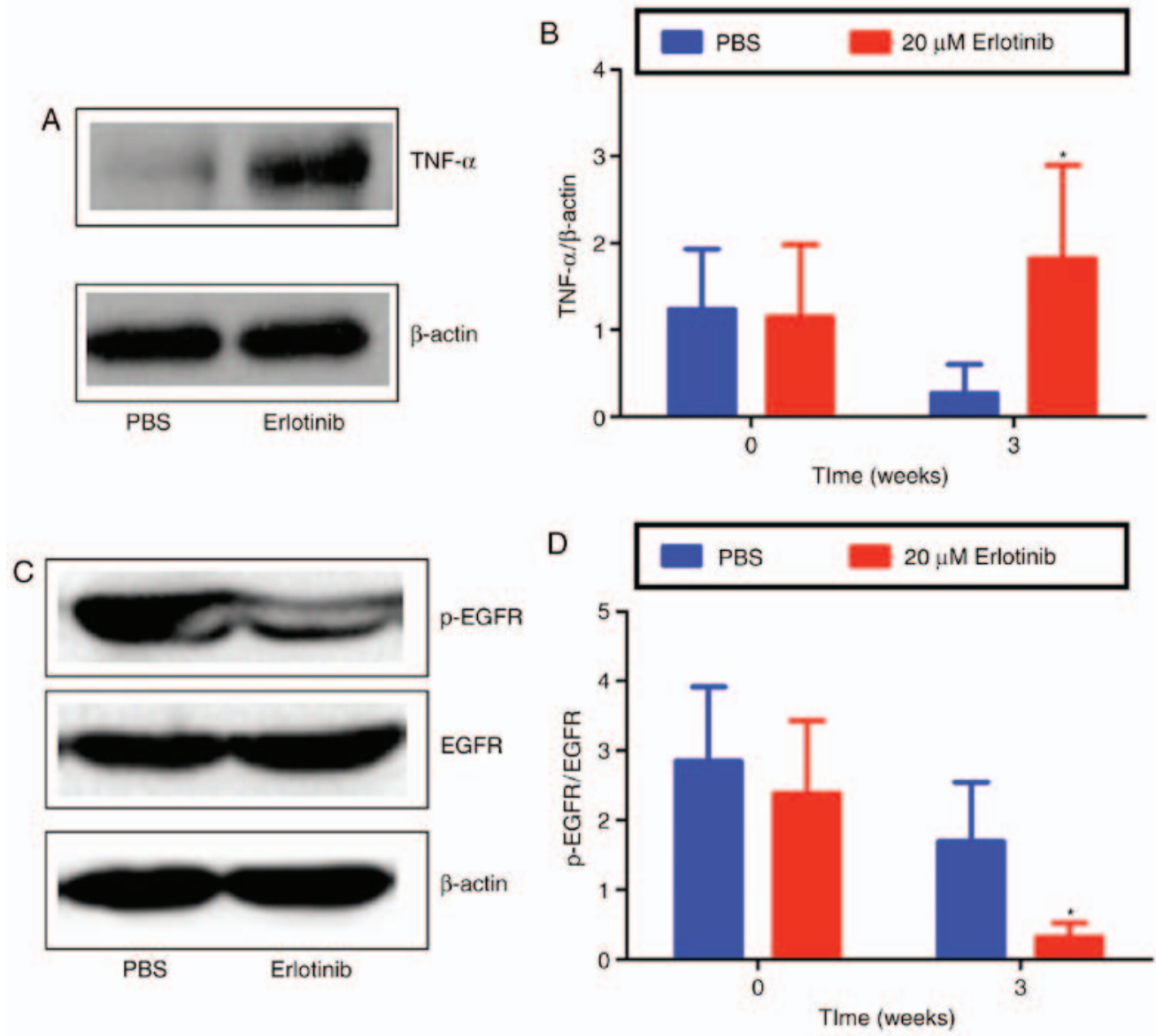

Figure 6. Effect of erlotinib on TNF- $\alpha$, EGFR and p-EGFR protein expression levels. (A) Following treatment for 3 weeks with either erlotinib or PBS, the protein expression levels of TNF- $\alpha$ were determined by western blot analysis, and the (B) density of the bands was statistically analyzed. (C) Following treatment for 3 weeks with either erlotinib or PBS, the protein expression levels of EGFR and p-EGFR were determined by western blot analysis, and the (D) density of the bands was used to statistically analyze the $\mathrm{p}$-EGFR/EGFR ratio. Data are presented as mean + standard deviation. ${ }^{*} \mathrm{P}<0.05$ vs. the PBS group at the same time point. TNF, tumor necrosis factor; EGFR, epidermal growth factor receptor; p, phosphorylated.

erlotinib-treated group at 3 weeks (Fig. $6 \mathrm{~A}$ and $\mathrm{B} ; \mathrm{P}<0.05$ ). The expression of EGFR remained similar in the PBS- and erlotinib-treated groups; however, the expression of p-EGFR was markedly decreased in the ocular surface following treatment with erlotinib (Fig. 6C). The level of p-EGFR/EGFR expression was revealed to be significantly reduced at 3 weeks in the erlotinib group compared with the level in the PBS group $(\mathrm{P}<0.05$; Fig. 6D). Together, the results suggest that topical erlotinib reduced EGFR activation and induced inflammation in the development of dry eyes.

\section{Discussion}

To the best of our knowledge, the present study is the first to investigate the effects of erlotinib eye drops on the ocular surface of mice. Erlotinib is a small molecule quinazoline compound; it is used as an anti-tumor drug that targets protein tyrosine kinase A. Erlotinib is used for the clinical treatment of NSCLC where it acts as a competitor for adenosine triphosphate (ATP) binding sites on the intracellular domain of tyrosine kinase (18). This consequently reversibly and selectively inhibits the phosphorylation of EGFR and the related tyrosine kinase activities, including the downstream signal transduction pathway to block angiogenesis, cell proliferation and tumor growth (19). In the present study, it was revealed that the expression of corneal p-EGFR in the erlotinib group was significantly lower than in the PBS group, which indicated that the activation of EGFR was inhibited.

In 2007, the International Dry Eye Working Group proposed that dry eyes should be defined as 'ocular symptoms caused by multiple factors, including tear film instability, changes of visual acuity and potential ocular surface damage, decreased tear osmolarity, and increased and ocular inflammation' (20). Dry eyes have become a global epidemic (21). According to epidemiological data, the worldwide prevalence is $\sim 35 \%$ (22). Age, environment, medicine and other factors have been identified as being associated with dry eye disease (23). Numerous drugs may cause dry eye disease, including EFGR inhibitor erlotinib used for the treatment of NSCLC (24). Previous papers that have reported the ocular side effects of erlotinib are listed in Table I.

Epidermal growth factor (EGF) has the ability to stimulate cell proliferation and differentiation, and promote tissue growth, development and maturation (25). EGF is a potent cytokine in mammalian eyes, and may stimulate the proliferation, chemoattraction and migration of conjunctival and corneal epithelial cells during wound healing (26). EGF serves an important role in the self-renewal of corneal epithelial cells and is required to maintain the stability of the corneal microenvironment (27). EGF binds with the EGFR to 


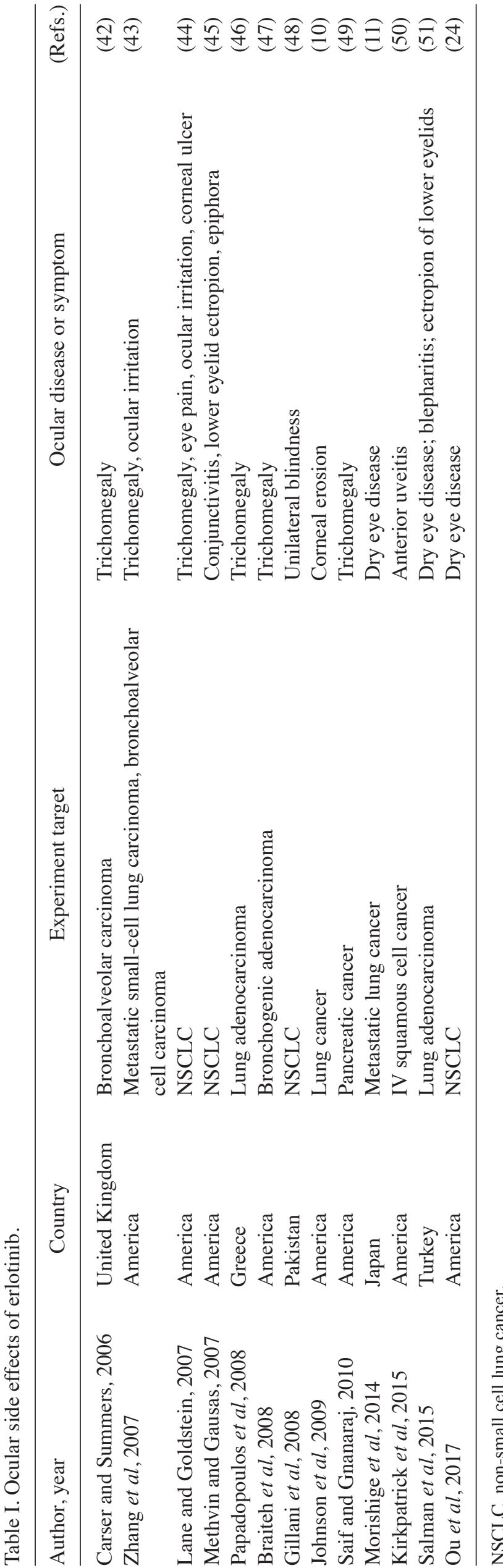

activate a series of signal transduction pathways, which induce protein phosphorylation and lead to the fast differentiation and proliferation of cells to accelerate the healing of corneal injuries (28). EFGR is expressed in the human cornea (29). EGF is abundant in tears and it may increase corneal epithelial cell proliferation (30). Corneal epithelial damage, inflammation, dry eye treatment drugs and reduced tear secretion may result in an insufficient level of EGF to enable corneal wound healing, which aggravates dry eye disease (31). In the present study, following 3 weeks of topical erlotinib administration, it was revealed that the dry eye-associated inflammatory index significantly increased, as well as sodium fluorescein and Lissamine green staining. Conversely, BUT and tear volume significantly decreased. The results demonstrated that erlotinib may cause ocular surface damage similar to dry eye disease. Histopathology revealed that the corneal epithelial cells in the experimental group had a disordered arrangement, the number of cell layers was increased and cell junctions were not clear. Additionally, there were cells shedding from the epithelium and a significantly higher number of vacuolated cells compared with the control group.

Mucin exists in the aqueous layer in the tear film to help maintain its stability (32). Cell apoptosis, decreased goblet cells and changes in mucin expression may cause instability of the tear film and increased tear osmolarity (33). This may activate a series of inflammatory responses, which increase the ocular surface epithelium damage resulting in the formation of a damaging cycle (33). In addition, previous studies have reported that EGF may promote the proliferation of goblet cells, thereby increasing the stability of tear film $(34,35)$. Through the topical application of erlotinib eye drops, the present study revealed that erlotinib reduced the number of goblet cells, which may lead to reduced mucin secretion in the goblet cells, causing dry eye disease. The increased expression of TNF- $\alpha$ that was observed in the present study also suggests the presence of ocular surface inflammation.

Apoptosis is a basic cellular phenomenon, which serves an important role in the removal of unnecessary or abnormal cells (36). Previous studies have reported that the apoptosis of conjunctival and corneal epithelial cells has a close association with dry eye disease $(15,37)$. Erlotinib acts directly on the ATP binding sites of the tyrosine kinase domain, which interferes with the binding of ATP with tyrosine kinase, thereby inhibiting the activity of tyrosine kinase and blocking the signaling pathway. Consequently, erlotinib inhibits the proliferation of cells and angiogenesis, resulting in cell apoptosis (25). The results of the present study also revealed that erlotinib caused an increase in the apoptosis of corneal epithelial cells.

Microvilli in the corneal and conjunctival epithelial cells are necessary for the localization of tear film on the subcellular structure of the ocular surface (38). Normal corneal epithelial cell surface microvilli and micro-structural folds are conducive for the corneal epithelial adhesion of the various components of the tear film, including mucin (MUC1, MUC4, MUC16) secreted by corneal epithelial cells (39). The interaction of corneal epithelial microvilli and membrane-bound mucin evenly coats the surface of the cornea with tears through blinking, and protects epithelial cells from any damage caused by the friction of the blink, which maintains non-keratinized characteristics of corneal epithelial cells $(40,41)$. When epithelial cell microvilli 
are abnormal in shape and structure, mucin may not be coated on the surface of corneal epithelial cells. Erlotinib may therefore inhibit the repair of corneal epithelial tissue and cell damage. Using an electron microscope, it was revealed that the number of corneal epithelial microvilli were significantly decreased in the erlotinib group compared with the control group, and that the ultrastructure of microvilli also had notable differences. Occasionally, digitations of microvilli were observed with the majority being short and irregularly arranged.

In conclusion, in the present study, the administration of topical erlotinib eye drops was demonstrated to cause histopathological and ultrastructural changes to the corneal epithelial cells, similar to dry eye disease in an animal model reported in a previous study (34). Whether erlotinib is the cause of dry eye disease, and the mechanism by which it has an effect, require further investigation.

\section{Acknowledgements}

The present study was supported by the National Natural Science Foundation of China (grant nos. 81160118, 81460092, 81660158 and 81400372).

\section{References}

1. Gansler T, Ganz PA, Grant M, Greene FL, Johnstone P, Mahoney M, Newman LA, Oh WK, Thomas CR Jr, Thun MJ, et al: Sixty years of CA: A cancer journal for clinicians. CA Cancer J Clin 60: 345-350, 2010.

2. Vijayvergia $\mathrm{N}$ and Mehra R: Clinical challenges in targeting anaplastic lymphoma kinase in advanced non-small cell lung cancer. Cancer Chemother Pharmacol 74: 437-446, 2014.

3. Zhou C, Wu YL, Chen G, Feng J, Liu XQ, Wang C, Zhang S, Wang J, Zhou S, Ren S, et al: Erlotinib versus chemotherapy as first-line treatment for patients with advanced EGFR mutation-positive non-small-cell lung cancer (OPTIMAL, CTONG-0802): A multicentre, open-label, randomised, phase 3 study. Lancet Oncol 12: 735-742, 2011.

4. Shepherd FA, Rodrigues Pereira J, Ciuleanu T, Tan EH, Hirsh V, Thongprasert S, Campos D, Maoleekoonpiroj S, Smylie M, Martins R, et al: Erlotinib in previously treated non-small-cell lung cancer. N Engl J Med 353: 123-132, 2005.

5. Verkhivker GM: Exploring sequence-structure relationships in the tyrosine kinome space: Functional classification of the binding specificity mechanisms for cancer therapeutics. Bioinformatics 23: 1919-1926, 2007.

6. Yin S, Zhou L, Lin J, Xue L and Zhang C: Design, synthesis and biological activities of novel oxazolo[4,5-g]quinazolin-2(1H)-one derivatives as EGFR inhibitors. Eur J Med Chem 101: 462-475, 2015.

7. Xiong X, Liu H, Fu L, Li L, Li J, Luo X and Mei C: Antitumor activity of a new $\mathrm{N}$-substituted thiourea derivative, an EGFR signaling-targeted inhibitor against a panel of human lung cancer cell lines. Chemotherapy 54: 463-474, 2008.

8. Dickler MN, Cobleigh MA, Miller KD, Klein PM and Winer EP: Efficacy and safety of erlotinib in patients with locally advanced or metastatic breast cancer. Breast Cancer Res Treat 115: 115-121, 2009.

9. Fraunfelder FT, Sciubba JJ and Mathers WD: The role of medications in causing dry eye. J Ophthalmol 2012: 285851, 2012.

10. Johnson KS, Levin F and Chu DS: Persistent corneal epithelial defect associated with erlotinib treatment. Cornea 28: 706-707, 2009.

11. Morishige N, Hatabe N, Morita Y, Yamada N, Kimura K and Sonoda KH: Spontaneous healing of corneal perforation after temporary discontinuation of erlotinib treatment. Case Rep Ophthalmol 5: 6-10, 2014.

12. Saint-Jean A, Sainz de la Maza M, Morral M, Morral M, Torras J, Quintana R, Molina JJ and Molina-Prat N: Ocular adverse events of systemic inhibitors of the epidermal growth factor receptor: Report of 5 cases. Ophthalmology 119: 1798-1802, 2012.
13. Barabino S, Chen W and Dana MR: Tear film and ocular surface tests in animal models of dry eye: Uses and limitations. Exp Eye Res 79: 613-621, 2004.

14. Romay C,Armesto J, Remirez D, González R, Ledon N and García I: Antioxidant and anti-inflammatory properties of C-phycocyanin from blue-green algae. Inflamm Res 47: 36-41, 1998.

15. Zhang Z, Yang WZ, Zhu ZZ, Hu QQ, Chen YF, He H, Chen YX and Liu ZG: Therapeutic effects of topical doxycycline in a benzalkonium chloride-induced mouse dry eye model. Invest Ophthalmol Vis Sci 55: 2963-2974, 2014.

16. Lemp MA: Report of the national eye institute/industry workshop on clinical trials in dry eyes. Clao J 21: 221-232, 1995.

17. Williams KA, Standfield SD, Smith JR and Coster DJ: Corneal graft rejection occurs despite Fas ligand expression and apoptosis of infiltrating cells. Br J Ophthalmol 89: 632-638, 2005.

18. Carey KD, Garton AJ, Romero MS, Kahler J, Thomson S, Ross S, Park F, Haley JD, Gibson N and Sliwkowski MX: Kinetic analysis of epidermal growth factor receptor somatic mutant proteins shows increased sensitivity to the epidermal growth factor receptor tyrosine kinase inhibitor, erlotinib. Cancer Res 66: 8163-8171, 2006.

19. Minna JD and Dowell J: Erlotinib hydrochloride. Nat Rev Drug Discov (Suppl): S14-S15, 2005.

20. The definition and classification of dry eye disease: Report of the Definition and Classification Subcommittee of the International Dry Eye WorkShop (2007). Ocul Surf 5: 75-92, 2007.

21. Stapleton F, Alves M, Bunya VY, Jalbert I, Lekhanont K, Malet F, Na KS, Schaumberg D, Uchino M, Vehof J, et al: TFOS DEWS II Epidemiology Report. Ocul Surf 15: 334-365, 2017.

22. Bose T, Diedrichs-Möhring M and Wildner G: Dry eye disease and uveitis: A closer look at immune mechanisms in animal models of two ocular autoimmune diseases. Autoimmun Rev 15: 1181-1192, 2016.

23. Liu NN, Liu L, Li J and Sun YZ: Prevalence of and risk factors for dry eye symptom in mainland china: A systematic review and meta-analysis. J Ophthalmol 2014: 748654, 2014.

24. Ou SI, Govindan R, Eaton KD, Otterson GA, Gutierrez ME, Mita AC, Argiris A, Brega NM, Usari T, Tan W, et al: Phase I results from a study of crizotinib in combination with erlotinib in patients with advanced nonsquamous non-small cell lung cancer. J Thorac Oncol 12: 145-151, 2017.

25. Wang L, Wu X, Shi T and Lu L: Epidermal growth factor (EGF)-induced corneal epithelial wound healing through nuclear factor $\kappa \mathrm{B}$ subtype-regulated CCCTC binding factor (CTCF) activation. J Biol Chem 288: 24363-24371, 2013.

26. Huo YN, Chen W and Zheng XX: ROS, MAPK/ERK and PKC play distinct roles in EGF-stimulated human corneal cell proliferation and migration. Cell Mol Biol (Noisy-le-grand) 61: 6-11, 2015.

27. Nakamura $\mathrm{Y}$, Sotozono $\mathrm{C}$ and Kinoshita S: The epidermal growth factor receptor (EGFR): Role in corneal wound healing and homeostasis. Exp Eye Res 72: 511-517, 2001.

28. Du H, Hu Z, Bazzoli A and Zhang Y: Prediction of inhibitory activity of epidermal growth factor receptor inhibitors using grid search-projection pursuit regression method. PLoS One 6: e22367, 2011.

29. Wilson SE, He YG and Lloyd SA: EGF, EGF receptor, basic FGF, TGF beta-1, and IL-1 alpha mRNA in human corneal epithelial cells and stromal fibroblasts. Invest Ophthalmol Vis Sci 33: 1756-1765, 1992

30. Kinoshita S, Adachi W, Sotozono C, Nishida K, Yokoi N, Quantock AJ and Okubo K: Characteristics of the human ocular surface epithelium. Prog Retin Eye Res 20: 639-673, 2001.

31. Bron AJ, de Paiva CS, Chauhan SK, Bonini S, Gabison EE, Jain S, Knop E, Markoulli M, Ogawa Y, Perez V, et al: TFOS DEWS II pathophysiology report. Ocul Surf 15: 438-510, 2017.

32. Ablamowicz AF and Nichols JJ: Ocular surface membrane-associated mucins. Ocul Surf 14: 331-341, 2016.

33. Stephens DN and McNamara NA: Altered mucin and glycoprotein expression in dry eye disease. Optom Vis Sc 92: 931-938, 2015.

34. Xiao X, He H, Lin Z, Luo P, He H, Zhou T, Zhou Y and Liu Z: Therapeutic effects of epidermal growth factor on benzalkonium chloride-induced dry eye in a mouse model. Invest Ophthalmol Vis Sci 53: 191-197, 2012.

35. Horikawa Y, Shatos MA, Hodges RR, Zoukhri D, Rios JD, Chang EL, Bernardino CR, Rubin PA and Dartt DA: Activation of mitogen-activated protein kinase by cholinergic agonists and EGF in human compared with rat cultured conjunctival goblet cells. Invest Ophthalmol Vis Sci 44: 2535-2544, 2003. 
36. Wickman G, Julian L and Olson MF: How apoptotic cells aid in the removal of their own cold dead bodies. Cell Death Differ 19: 735-742, 2012.

37. Yang L, Sui W, Li Y, Qi X, Wang Y, Zhou Q and Gao H: Substance P inhibits hyperosmotic stress-induced apoptosis in corneal epithelial cells through the mechanism of akt activation and reactive oxygen species scavenging via the neurokinin-1 receptor. PLoS One 11: e0149865, 2016.

38. Mackay M, Williamson I and Hastewell J: (A) Cell biology of epithelia. Adv Drug Deliver Rev 7: 313-338, 1991.

39. Schrader S, Notara M, Beaconsfield M, Tuft SJ, Daniels JT and Geerling G: Tissue engineering for conjunctival reconstruction: Established methods and future outlooks. Curr Eye Res 34 913-924, 2009.

40. Hori Y, Nishida K, Yamato M, Sugiyama H, Soma T, Inoue T, Maeda N, Okano T and Tano Y: Differential expression of MUC16 in human oral mucosal epithelium and cultivated epithelial sheets. Exp Eye Res 87: 191-196, 2008.

41. Yañez-Soto B, Mannis MJ, Schwab IR, Li JY, Leonard BC, Abbott NL and Murphy CJ: Interfacial phenomena and the ocular surface. Ocul Surf 12: 178-201, 2014.

42. Carser JE and Summers YJ: Trichomegaly of the eyelashes after treatment with erlotinib in non-small cell lung cancer. J Thorac Oncol 1: 1040-1041, 2006.

43. Zhang G, Basti S and Jampol LM: Acquired trichomegaly and symptomatic external ocular changes in patients receiving epidermal growth factor receptor inhibitors: case reports and a review of literature. Cornea 26: 858-860, 2007.
44. Lane K and Goldstein SM: Erlotinib-associated trichomegaly. Ophthal Plast Reconstr Surg 23: 65-66, 2007.

45. Methvin AB and Gausas RE: Newly recognized ocular side effects of erlotinib. Ophthal Plast Reconstr Surg 23: 63-65, 2007.

46. Papadopoulos R, Chasapi V and Bachariou A: Trichomegaly induced by erlotinib. Orbit 27: 329-330, 2008.

47. Braiteh F, Kurzrock R and Johnson FM: Trichomegaly of the eyelashes after lung cancer treatment with the epidermal growth factor receptor inhibitor erlotinib. J Clin Oncol 26: 3460-3462, 2008.

48. Gillani JA, Iqbal M, Nasreen S, Khan A and Samad A: Unilateral blindness as a presenting symptom of lung cancer treated with erlotinib. J Coll Physicians Surg Pak 18: 125-126, 2008.

49. Saif MW and Gnanaraj J: Erlotinib-induced trichomegaly in a male patient with pancreatic cancer. Cutan Ocul Toxicol 29: 62-66, 2010.

50. Kirkpatrick CA, Almeida DR, Hornick AL, Chin EK and Boldt HC: Erlotinib-associated bilateral anterior uveitis: Resolution with posterior sub-Tenon's triamcinolone without erlotinib cessation. Can J Ophthalmol 50: e66-e67, 2015.

51. Salman A, Cerman E, Seckin D and Kanitez M: Erlotinib induced ectropion following papulopustular rash. J Dermatol Case Rep 9: 46-48, 2015. 